\title{
Electronic relaxation in Ag nanoclusters studied with time-resolved photoelectron spectroscopy
}

\author{
M. Niemietz, ${ }^{1}$ M. Engelke, ${ }^{1}$ Y. D. Kim, ${ }^{2}$ and G. Ganteför ${ }^{1}$ \\ ${ }^{1}$ Department of Physics, University of Konstanz, D-78457 Konstanz, Germany \\ ${ }^{2}$ Division of Nano Sciences and Department of Chemistry, Ewha Womans University, 120-750 Seoul, Korea
}

\begin{abstract}
The decay mechanism of excited electronic states in small Ag nanoclusters is studied using time-resolved photoelectron spectroscopy. The low density of states in these clusters inhibits Auger-like decay channels that are responsible for ultrafast relaxation of optically excited states in bulk Ag. Thus, much longer relaxation times are expected for small clusters showing large gaps between electronic states. In contrast to this expectation, lifetimes below 1 ps were observed for most of the $\mathrm{Ag}_{n}^{-}(n<22)$ studied here. The only exception is the magic $\mathrm{Ag}_{7}^{-}$with a relaxation time of $3.8 \mathrm{ps}$. The observed fast relaxations are discussed in view of their ability to undergo fast shape deformations. This approach can also explain the slow relaxation of the rigid $\mathrm{Au}_{n}^{-}$ nanoclusters and the extremely fast relaxation of the flexible magic $\mathrm{Al}_{13}^{-}$.
\end{abstract}

PACS number(s): 61.46.Bc, 73.22.-f, 78.47.+p, 79.60.-i

\section{INTRODUCTION}

Behaviors of nanoclusters in many aspects differ from those of the corresponding bulk materials. One major issue about the unique properties of clusters is the electronic relaxation after photoexcitation. Experimentally, this can be explored using time-resolved photoelectron spectroscopy: a pump photon excites a single electron from an occupied level to an unoccupied state, and the subsequent development of the electronic structure is monitored with a second photon recording a photoelectron spectrum. ${ }^{1-8}$ The spectrum mirrors the occupied states and it is possible to follow the path of the system on its way back to the ground state.

In bulk metals with a continuum of occupied and unoccupied states close to the Fermi energy, the relaxation occurs via Auger-like electron-electron scattering processes. ${ }^{9-12}$ The energy, which is first transferred to a single electron, is dissipated within 1-100 fs over many electrons resulting in a thermal distribution of hot electrons. On a picosecond (ps) time scale, this energy is transferred to the phonon degrees of freedom. Within this model, the fast relaxation within femtoseconds (fs) is not likely in small clusters of $s$ metals such as $\mathrm{Ag}$, because there are no unoccupied orbitals located close to the Fermi level [now called the highest occupied molecular orbital (HOMO)]. Thus, low energy electronic excitations are not possible. Therefore, when the average distance between two neighboring levels is comparable to the photon energy (here, $\sim 1.5 \mathrm{eV}$ ), Auger processes, which dominate relaxations in bulk metals, can be neglected.

An alternative bulk decay channel is phonon-assisted relaxation, in which a radiationless transition results in the excitation of phonon modes. However, if the electronic level spacing is much larger than the typical energy of a phonon, excitation of many phonon modes should take place for the relaxation of an optically excited state, which is rather unlikely. This problem is well known for semiconductor nanoparticles and referred to as the "phonon bottleneck." 13 As a result, rather long lifetimes are to be expected and the optically excited states in clusters may only decay via photon emission on nanosecond time scale.

Previously, we indeed observed such a behavior for certain Au clusters. ${ }^{3,7}$ Excited states of the anions $\mathrm{Au}_{3}^{-}$and $\mathrm{Au}_{6}^{-}$ with $1.5 \mathrm{eV}$ excitation energy have lifetimes in the nanosec- ond regime in agreement with the considerations mentioned above. However, an electron hole excitation of $\mathrm{Al}_{13}^{-}$with an energy almost exactly matching the gap between HOMO and lowest unoccupied molecular orbital (LUMO) relaxes within 200 fs down to the ground state, which cannot be understood with the relaxation mechanism discussed above.

Nanoclusters consisting of less than 100 atoms have another degree of freedom different from bulk and large nanoparticles: they can undergo fast structural changes as a response to excitations. During such a geometric transformation the single particle orbitals continuously change their binding energies and might even cross in the energy diagram. This concept, which is referred to as internal conversion, is commonly used for molecules and describes the transition of an excited state to the ground state via a level crossing induced by a change of the geometric structure. ${ }^{14-16}$ The potential surface of the ground and excited states might touch in a particular geometry during the deformation process, resulting in a fast and efficient relaxation of the excited state. The crossing point in the energy diagram is described as a conical intersection. The concept of internal conversion provides an explanation for the fast relaxation observed in the case of $\mathrm{Al}_{13}^{-}$, as pointed out by Kresin et al. ${ }^{17} \mathrm{It}$ is interesting to note that recently an excitation induced lattice relaxation is also considered as a solution of the "phonon bottleneck" problem. ${ }^{13}$

In order to check the validity of the ideas aforementioned for other clusters, we here studied the electronic relaxation in size-selected $\mathrm{Ag}_{n}^{-}$. In addition to the alkali metals, which are more difficult to handle, $\mathrm{Ag}$ is the one metal with electronic properties most similar to a Fermi liquid. ${ }^{12}$ Among the coinage metals Ag clusters can be seen as the best example for the electronic shell model with the least influence of $d$ electrons. ${ }^{18}$ Ag clusters show a pronounced even-odd alternation of the electron affinities and electronic shell closings at 8 and 20 electrons. ${ }^{19}$ The geometric and electronic structures of the anions have been studied in many experiments, especially using photoelectron spectroscopy in comparison with theory. ${ }^{20-22}$ First results on the dynamics of $\mathrm{Ag}_{3}^{-}$were obtained in a pioneering $\mathrm{NeNePo}$ study, where the rapid change of geometry after photodetachment has been measured indicating a rather fluxional geometry of the trimer. ${ }^{23}$ For neutral $\mathrm{Ag}_{8}$ a very narrow absorption resonance has been 
found and even fluorescence in an Argon matrix was observed after excitation. ${ }^{24,25}$ The existence of a long-living state for this cluster is also indicated by the results of a two-photon photoionization experiment. ${ }^{26}$

Using time-resolved photoelectron spectroscopy we found rather short relaxation times of less than $1 \mathrm{ps}$ for most $\mathrm{Ag}_{n}^{-}$, analogous to the results of $\mathrm{Al}_{n}^{-}$. We explain these findings by the larger structural flexibility of Ag clusters compared to $\mathrm{Au}$ clusters, with rather rigid structures stabilized by relativistic effects. Only $\mathrm{Ag}_{7}^{-}$, one of the magic clusters with a large gap between electronic states exhibits a relaxation time of about $4 \mathrm{ps}$, which agrees with the earlier indications for the existence of a long-living state in an eight-electron $\mathrm{Ag}$ cluster but is still much faster than for $\mathrm{Au}_{6}^{-}$. Our new results on $\mathrm{Ag}_{n}^{-}$in comparison with the earlier findings for $\mathrm{Al}_{n}^{-}$and $\mathrm{Au}_{n}^{-}$can be qualitatively understood by the competition of three decay channels: Auger decay, relaxation via vibrational modes (corresponding to phonon-assisted relaxation in the bulk) and internal conversion. ${ }^{6,7}$ Excitation-induced fast deformations seem to be a dominant decay mechanism enabling fast energy dissipation in clusters.

\section{EXPERIMENTAL SETUP}

The experimental setup has been discussed in detail elsewhere. ${ }^{6-8}$ Briefly, Ag cluster anions are synthesized using a pulsed arc cluster ion source (PACIS) and mass selected using time-of-flight mass spectrometry. After mass selection, the clusters are irradiated by two laser pulses generated by a femtosecond laser system. The first pulse has a photon energy of $1.55 \mathrm{eV}$ and the second one a photon energy of $3.1 \mathrm{eV}$. The pump pulse can excite a fraction of the anions in the mass-selected bunch whereas the probe pulse detaches electrons from the same bunch. The photon flux of both pulses is optimized in order to minimize the number of cluster anions hit by two or more photons from the same pulse, and to maximize the number of species hit by one single photon from each pulse. The photon flux can be optimized with the following procedure: only using the pump pulse the photon flux is decreased below a certain value showing onset of electron detachment by two-photon processes. The same procedure can be applied for the probe pulse. At the stage of two-photon photoemission spectroscopy, the cluster temperature is estimated to be roughly room temperature. ${ }^{27}$

The experimental time-resolution is determined by recording pump-probe spectra of a short-living excited state. As an example, Fig. 1 displays a series of pump-probe photoelectron spectra of $\mathrm{Ag}_{3}^{-}$. The pump-probe signal at a kinetic energy of $2 \mathrm{eV}$ indicates the existence of an excited state with a lifetime much shorter than our time resolution. The intensity of this peak plotted versus pump-probe delay corresponds to the crosscorrelation curve and gives the real time-resolution of our apparatus (110 fs). In addition, we take the maximum of this signal as the time zero. If the (unknown) lifetime of the excited state is not negligible, the time resolution measured in this way gives an upper limit of the real time resolution.

\section{RESULTS}

The photon energy of the pump-pulse cannot be varied in our experimental system. Therefore, we can only observe a

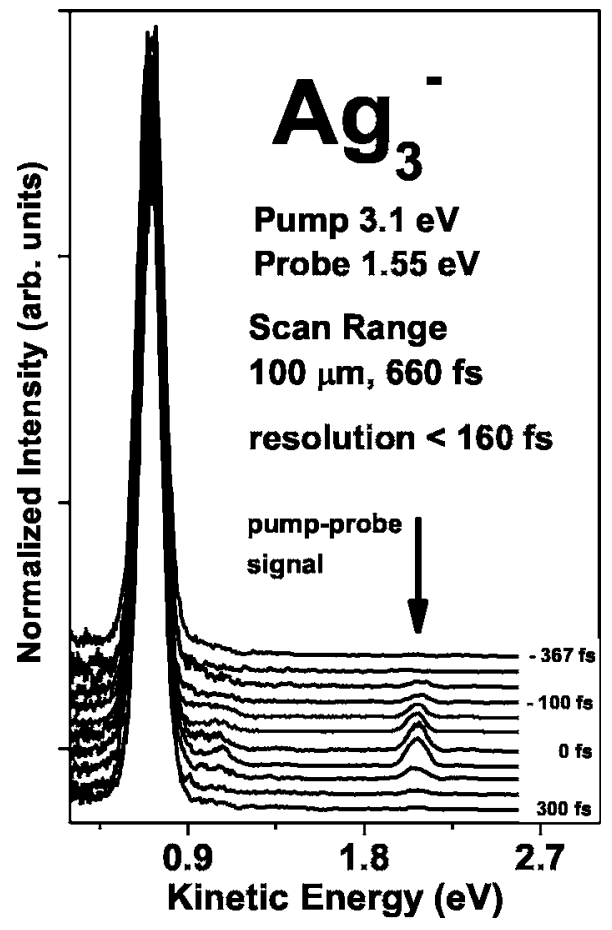

FIG. 1. A series of the pump-probe photoelectron spectra of $\mathrm{Ag}_{3}^{-}$ is displayed proving the time-resolution of the experimental set up. The pump-probe signal exhibits the signature of an excited state with a lifetime much shorter than the time resolution of our experimental setup. In this case the dependence of the pump-probe signal on the delay between pump and probe pulse corresponds to the time resolution. The maximum is taken as time zero. The spectra are normalized to the photoelectron peak at a kinetic energy of $0.7 \mathrm{eV}$ corresponding to photodetachment from $\mathrm{Ag}_{3}^{-}$by one photon of the probe pulse. The 11 traces represent a scan range of 100 micrometers of the retroreflector corresponding to $660 \mathrm{fs}$. As time resolution (110 fs) we take the FWHM (160 fs) of the intensity of the pumpprobe signal plotted as a function of the pump-probe delay multiplied by 0.707 .

pump-probe signal for a cluster having an available excited state for the electron excitation using this fixed energy. We were able to detect such pump-probe signals for $\mathrm{Ag}_{n}^{-}$with $n=3,7-9,11,14,15,18,19$, and 21. In general, the amounts of $\mathrm{Ag}_{n}^{-}$generated by our source are rather small and in most cases the signal to noise ratios in the spectra did not allow detailed analysis of spectral features, which may provide indications of the relaxation paths. Only for the closed shell species $\mathrm{Ag}_{7}^{-}$and $\mathrm{Ag}_{19}^{-}$the pump-probe signals were sufficiently intense to shed some light onto the relaxation paths. For all clusters showing pump-probe signals, the timedependent signal intensity has been integrated and plotted versus pump-probe delay, resulting in an estimation of the relaxation times of the excited states (Fig. 2 and Table I) ${ }^{28}$ In most cases the time-dependent signal decreases, following an exponential law described by a single time constant. In other cases $(n=7,18,19)$ it is possible to distinguish between a fast and a slow component. Both values are listed in Table I. However, the true relaxation behavior might be more complex and the time constants extracted from our photoelectron spectra can only be taken as crude estimation, describing the behavior of the excited cluster. 


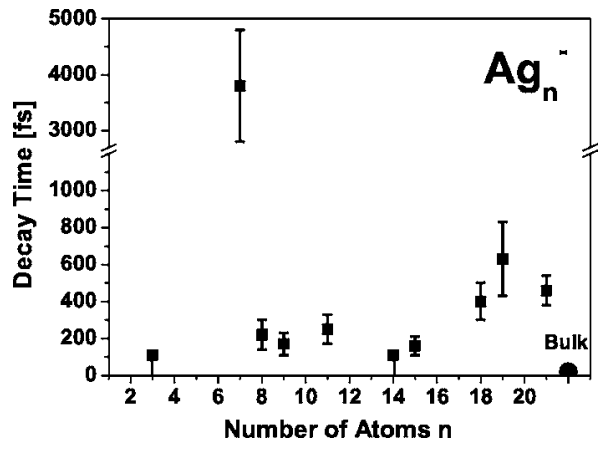

FIG. 2. The relaxation times (Ref. 28) listed in Table I (fast component only) plotted vs cluster size. The bulk value (Ref. 12) averaged for energies between $0.5 \mathrm{eV}$ and $1.5 \mathrm{eV}$ above the Fermi energy is also given.

Figure 3 displays a series of pump-probe photoelectron spectra of the magic cluster $\mathrm{Ag}_{7}^{-}$. The spectra are normalized to the one-photon emission peak (not shown) at $E_{\text {kin }}$ $=0.54 \mathrm{eV}$ caused by the probe photons corresponding to the known vertical detachment energy of this cluster, which is $2.56 \mathrm{eV} .{ }^{22}$ At zero delay a maximum at the expected position of $2.09 \mathrm{eV}=3.1+1.55-2.56 \mathrm{eV}$ appears. At long delays (trace recorded at $25.7 \mathrm{ps}$ ) the pump-probe signal vanishes. In between it exhibits a rather complex behavior with a change in shape, position, and intensity. The poor signal to noise ratio does not allow a profound discussion of shape variations and the gradual shift of its position. The timedependent signal was integrated between 1.2 and $2.5 \mathrm{eV}$ in kinetic energy for each spectrum. Since the observed de-

TABLE I. Relaxation times ${ }^{28}$ of the pump-probe signals of $\mathrm{Ag}_{n}^{-}$ after excitation by a $1.55 \mathrm{eV}$ photon. The relaxation times have been obtained by integrating the true pump-probe signal. These intensities decrease with increasing delay and the relaxation times are determined by a standard exponential fit procedure. If the relaxation time is equal to our experimental resolution (assuming Gaussian pulse shape), we cannot determine the relaxation time and $110 \mathrm{fs}$ is given as an upper limit. In some cases a slow and a fast component have been identified and both values are given. The value for bulk $\mathrm{Ag}$ averaged for excitation energies between $0.5 \mathrm{eV}$ and $1.5 \mathrm{eV}$ above Fermi energy is taken from Ref. 12.

\begin{tabular}{ccccc}
\hline \hline $\begin{array}{c}\text { Number } \\
\text { of atoms } \\
n\end{array}$ & $\begin{array}{c}\text { Relaxation time } \\
\text { fast component } \\
\mathrm{fs}\end{array}$ & $\begin{array}{c}\text { Error } \\
\pm \mathrm{fs}\end{array}$ & $\begin{array}{c}\text { Relaxation time } \\
\text { slow component } \\
\text { fs }\end{array}$ & $\begin{array}{c}\text { Error } \\
\pm \mathrm{fs}\end{array}$ \\
\hline 3 & $<110$ & & & \\
7 & 3800 & 1000 & 9500 & 2500 \\
8 & 220 & 80 & & \\
9 & 170 & 60 & & \\
11 & 250 & 80 & & \\
14 & $<110$ & & & \\
15 & 160 & 50 & & \\
18 & 400 & 100 & 2900 & \\
19 & 630 & 200 & 2700 & \\
21 & 420 & 80 & & \\
Bulk & 20 & 5 & & \\
\hline \hline
\end{tabular}

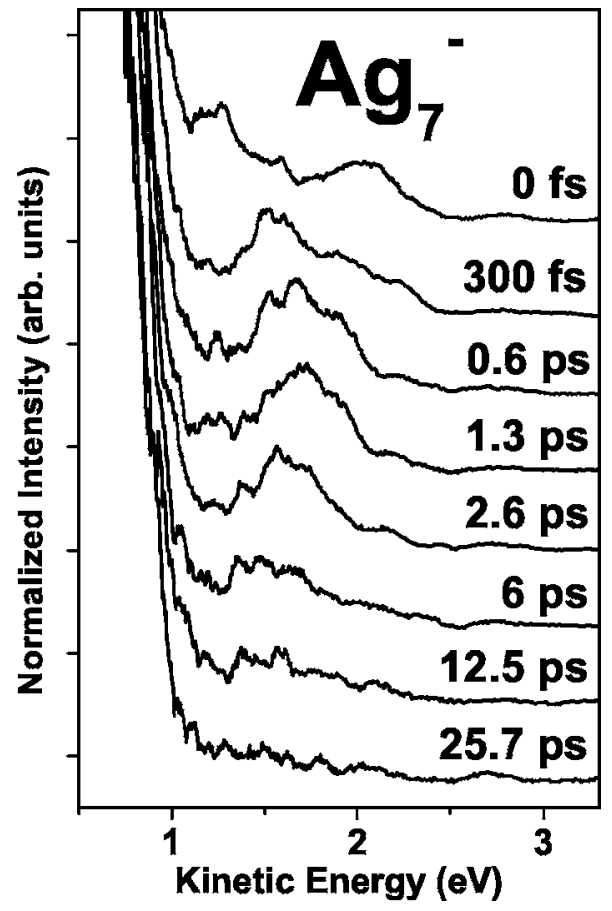

FIG. 3. A series of the pump-probe photoelectron spectra of $\mathrm{Ag}_{7}^{-}$ is displayed. The vertical detachment energy of $\mathrm{Ag}_{7}^{-}$is $2.56 \mathrm{eV}$ and the spectra are normalized to the signal at $E_{\text {kin }}=0.54 \mathrm{eV}$ (not shown). At zero delay a feature at $E_{\text {kin }}=2.1 \mathrm{eV}$ appears. With increasing delay this feature exhibits a rather complex behavior and finally disappears. The decrease in intensity is best fitted with two subsequent exponential decay functions with a time constant of $3.8 \mathrm{ps} \pm 1$ ps for delays $<3$ ps and $9.5 \mathrm{ps} \pm 2.5$ ps for longer delays.

crease in intensity was found to be fast within the first 3 ps and much slower thereafter, the curve was fitted by two subsequent exponential decay functions. The time constants for the decays are 3.8 and $9.5 \mathrm{ps}$, respectively (Table I). Obviously, the real relaxation process is rather complex and the description by two decay times is poor.

Figure 4 displays a series of pump-probe photoelectron spectra of $\mathrm{Ag}_{19}^{-}$. The spectra displayed are difference spectra, i.e., the delay-independent part of each photoelectron spectrum has been subtracted. The background spectrum was obtained by recording a spectrum at large delay, where no further change of the photoelectron spectrum can be observed. At zero delay a peak appears at $1.8 \mathrm{eV}$ corresponding to the excitation of an electron of the uppermost occupied orbital of the anion $(2 s)$ by a photon of the pump pulse. With increasing delay the pump-probe feature exhibits a gradual shift towards higher binding energy developing into a maximum between $1.1-1.5 \mathrm{eV}$ in kinetic energy at $1.3 \mathrm{ps}$. This feature disappears with further increasing delay. Again, the relaxation can be described by two time constants: the signal in the interval between 1.5 and $2.0 \mathrm{eV}$ decreases with a time constant of $630 \mathrm{fs}$, while the broad peak between $1.1 \mathrm{eV}-1.5 \mathrm{eV}$ vanishes more slowly with a time constant of $2.7 \mathrm{ps}$.

\section{DISCUSSIONS}

None of the $\mathrm{Ag}_{n}^{-}$studied here exhibits a relaxation time comparable to those of $\mathrm{Au}_{3}^{-}$or $\mathrm{Au}_{6}^{-}$. Auger-like processes and 


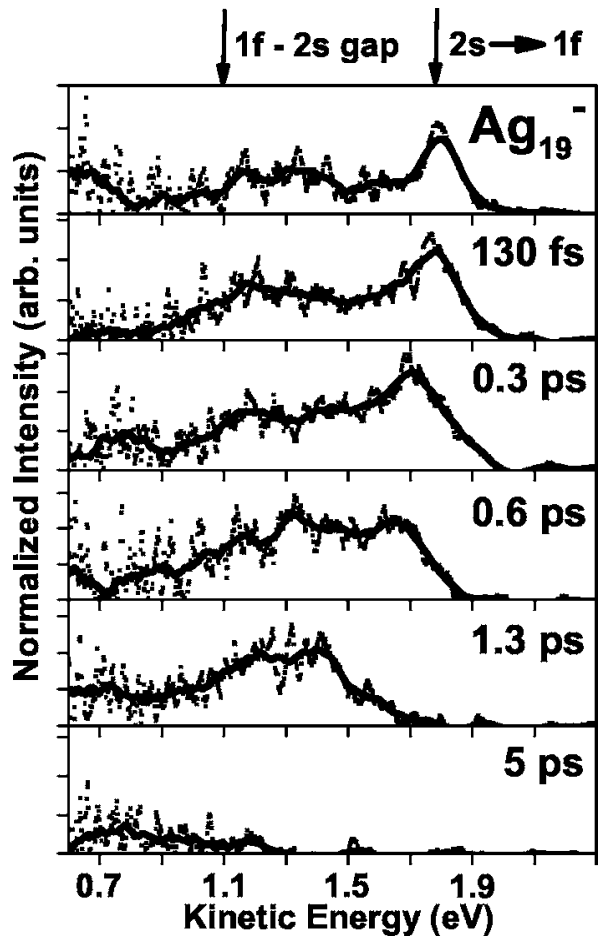

FIG. 4. A series of time-resolved photoelectron spectra of $\mathrm{Ag}_{19}^{-}$ is displayed. Only the time-dependent part of the photoelectron signal is shown. The delay-independent signal, which has been determined by recording a spectrum at a large delay when no further detectable changes occur, has been subtracted. At zero delay a peak appears at the expected position of the pump-probe signal as calculated from the sum of the photon energies minus the vertical detachment energy (indicated by an arrow: $1.82 \mathrm{eV}=1.55+3.1$ $-2.83 \mathrm{eV}$ ). The estimated position of the upper edge of the $1 f / 2 s$ gap is indicated by a second arrow at $1.1 \mathrm{eV}$.

phonon assisted relaxation cannot rationalize the observed fast relaxation times in the present work because of the large energy gaps between electronic levels of these small clusters. Thus, as a possible decay mechanism, one should consider the high ability of clusters to deform as a response to a single electron excitation which might result in a very fast radiationless decay by internal conversion.

A qualitative understanding of our data on Ag cluster anions can be obtained within the following concept: $\mathrm{Ag}_{n}$ are mostly bound by their s-electrons while the contribution from $d$ electrons to the chemical bonds is small. ${ }^{18} s / p$ orbitals form bonds with less preference for certain bond angles or geometries compared to $d$ orbitals. Therefore, electronic excitations might easily induce a considerable change in the cluster geometry as indicated by the Nilsson diagram (Fig. 5): according to Fig. 5, a 20-electron cluster (e.g., $\mathrm{Ag}_{19}^{-}$) has the closed shell configuration $1 s^{2} 1 p^{6} 1 d^{10} 2 s^{2}$ and a spherical symmetry. After single electron excitation corresponding to the transition of a $2 s$ electron into the $1 f$ shell, the system gains binding energy by prolate deformation. Already for medium deformation the $1 f$ and $2 s$ sublevels cross and a radiationless relaxation is possible.

Originally, this approach has first been used by Kresin et $a l$. to explain the fast relaxation of the HOMO-LUMO exci-

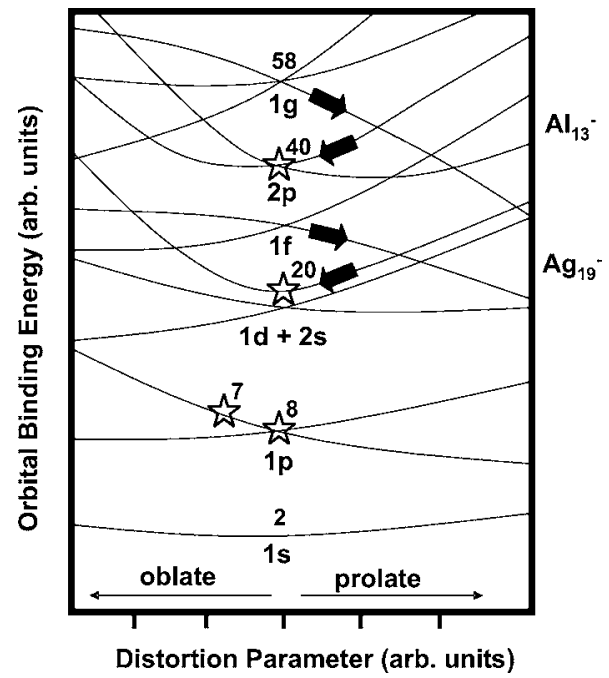

FIG. 5. The simplified Nilsson diagram (Ref. 19) of the electronic shells in clusters of simple metals is illustrated. Each curve corresponds to a non-degenerate single particle orbital in a deformed spherical potential. The binding energy depends on the degree of structural deformation. For simplification, only prolate and oblate deformations are considered and some sublevels have been omitted. At spherical symmetry the deformation parameter is zero and the sublevels are degenerate. The stars indicate the positions of the $7,8,20$, and 40 electron clusters. The configuration of a 20 electron cluster (e.g., $\mathrm{Ag}_{19}^{-}$) is $1 s^{2} 1 p^{6} 1 d^{10} 2 s^{2}$ and the lowest possible excitation corresponds to a $2 s \rightarrow 1 f$ transition. Analogous, a 40 electron cluster (e.g., $\mathrm{Al}_{13}^{-}$) undergoes a $2 p \rightarrow 1 g$ excitation. For both cases possible radiationless relaxation pathways are indicated by arrows.

tation in $\mathrm{Al}_{13}^{-}$, which was observed by our group. ${ }^{17}$ They showed theoretically that nanoclusters can support a fast nonradiative relaxation channel which derives from their distinctive ability to undergo Jahn-Teller shape deformations. The calculations were done for a three-dimensional potential well undergoing a spheroidal distortion and the timeevolution of the distortion parameter is calculated. The basic concept of these calculations, internal conversion via a conical intersection, is illustrated in Fig. 5 and possible relaxation pathways are indicated by arrows.

In the case of $\mathrm{Au}_{n}$, the geometric structure is strongly influenced by the $d$ electrons and the large spin-orbit interactions. ${ }^{29}$ For these reasons $\mathrm{Au}_{n}^{-}$anions up to $n \sim 14$ are planar. ${ }^{30}$ Here, an electronic excitation might yield a weaker geometric change and the mechanism of internal conversion is not valid because of too rigid geometric structures. This explains the extremely long lifetimes of some excited states of $\mathrm{Au}_{n}^{-}$.

The picture of purely $s / p$ bonded flexible clusters, which undergo considerable geometrical changes after electronic excitations, explains the short relaxation times for $\mathrm{Ag}_{n}^{-}$(Fig. 2 and Table I). Among the species studied here, $\mathrm{Ag}_{3}^{-}$shows a very fast relaxation of the excited state. This can also be understood in terms of a fast shape deformation. The ground state $\mathrm{Ag}_{3}^{-}$anion is linear, while the neutral and positively charged trimers are triangular. An excited state of $\mathrm{Ag}_{3}^{-}$might also be triangular, so that photoexcitation of the linear trimer 
anion might trigger a fast geometry change, during which the system passes through a crossing point with the ground state potential surface and radiationless relaxation occurs. In the already mentioned NeNePo study of the trimer a slower dynamical response time $(700 \mathrm{fs})$ was observed. ${ }^{23}$ The time scale depends on the local gradient of the potential energy surface of the final state and might be different for different processes and different excitation energies in the same system.

$\mathrm{Ag}_{7}^{-}$is the only exception of the general behavior with a lifetime of about an order of magnitude longer than those of all other $\mathrm{Ag}_{n}^{-}$. It has the largest HOMO-LUMO gap among the clusters studied here, ${ }^{19-22}$ which might explain the longer relaxation time. However, it is important to note that $\mathrm{Al}_{13}^{-}$has a gap of similar size but exhibits an extremely short relaxation time. ${ }^{6}$ Hence, a large gap alone does not explain our data on $\mathrm{Ag}_{7}^{-}$. Based on the Nilsson diagram in Fig. 5, the longer lifetime of the excited state in $\mathrm{Ag}_{7}^{-}$compared to $\mathrm{Al}_{13}^{-}$ can be justified: in the case of the eight-electron cluster a much larger geometrical deformation is necessary than for a cluster with 40 electrons $\left(\mathrm{Al}_{13}^{-}\right)$to approach a crossing point. The series of photoelectron spectra (Fig. 3) show that the excited $\mathrm{Ag}_{7}^{-*}$ immediately starts to change its geometry resulting in a fast initial shift of the excited state towards lower energy. It then needs a relatively long time for further deformation before the cluster finally approaches a crossing point and the peak in Fig. 3 disappears. Thus, the longer time scale of relaxation observed for $\mathrm{Ag}_{7}^{-}$(Fig. 3) can be related to the large deformation necessary for a crossing of the $1 d$ and $1 p$ sublevels in Fig. 5 as well as the large HOMO-LUMO gap. Our finding of a significantly enhanced relaxation time of the 8 electron $\mathrm{Ag}_{7}^{-}$agrees well with the results on neutral $\mathrm{Ag}_{8}$ mentioned above, indicating the existence of a long-living excited state for this magic cluster. ${ }^{24-26}$

For most of the $\mathrm{Ag}_{n}^{-}$relaxation after photoexcitation might proceed via a combination of various mechanisms involving Auger-like processes, relaxation via vibrational modes and internal conversion. The relaxation times become larger by about a factor of 2 for clusters with 18 atoms or more (Fig. 2 and Table I). The larger clusters-especially around the shell closings at 18 and 20 electrons-might have a stronger preference for a spherical geometry and an excitation involving the $2 s$ orbital might induce only a small change of the cluster geometry. This could explain the slower relaxations of theses clusters, i.e., although relaxations via other mechanisms might be enhanced due to the higher density of states, ultrafast relaxation might be slower for the larger clusters in the size regime between $n=18-21$.

For $\mathrm{Ag}_{19}^{-}$the signal to noise ratio is sufficiently high to analyze the structures observed in the time resolved photoelectron data (Fig. 4). The maximum of the pump probe signal shifts gradually from $1.8 \mathrm{eV}$ in kinetic energy to about $1.2 \mathrm{eV}$ and then disappears. Again, this behavior can be explained by a structural deformation of the cluster after photoexcitation with a final radiationless internal conversion back to the ground state. However, if we assume a rigid geometric structure of this cluster, an alternative interpretation of the spectra is possible. The excited electron first moves within the manifold of levels of the electronic shell above the $2 s$ level (= the $1 f$ orbital containing seven subor- bitals) similar to the relaxation of an excited electron in the conduction band of a semiconductor, and then approaches the lowest edge of the $1 f$ manifold of states above the $2 s-1 f$ gap. The broad maximum visible in the spectrum taken at 1.3 ps delay is located right above this gap between the $2 s$ shell and the $1 f$ manifold of states. The size of this gap can be estimated from a careful analysis of photoelectron spectra of $\mathrm{Ag}_{n}^{-}$taken at higher photon energy. ${ }^{22}$ Such a relaxation via electron-phonon-like interactions is possible, if the level spacing is comparable to the typical energy of the vibrational modes.

According to the considerations mentioned above, we propose internal conversion as the dominant relaxation channel of excited states in metal clusters with low density of electronic states and a fluxional geometry. The deformation of a cluster induced by photoexcitation of a single electron can be visualized using the Nilsson diagram. The simplified Nilsson diagram displayed in Fig. 5 shows that already a small change in the geometry of a 40 electron cluster results in closure of the $1 g-2 p$ gap. ${ }^{17}$ Thus, most likely the deformation leads to a conical intersection and internal conversion, during which the excess energy is converted to heat. In Fig. 5 one possible relaxation path of an excited state of $\mathrm{Al}_{13}^{-}$ is indicated by arrows. However, one should note that the Nilsson diagram is a crude approximation. In a more accurate description, both the ground state and the excited state of a cluster have to be described by potential energy surfaces in a multidimensional space.

\section{CONCLUSION}

In summary, time resolved photoelectron spectroscopy was used to study the dynamics of size-selected $\mathrm{Ag}_{n}^{-}$with $n=3,7-9,11,14,15,17-19$, and 21 . The clusters are photoexcited by absorption of a single photon with an energy of $1.55 \mathrm{eV}$ and the time-dependent development of the excited system is observed by recording photoelectron spectra using a second laser pulse with a photon energy of $3.1 \mathrm{eV}$. For most of the species the excited state signal disappears on a time scale considerably shorter than 1 ps. Since the spacing between neighboring electronic levels is of the same order as the excitation energy and much larger than the typical energy of vibrational modes, relaxations via combinations of Augerlike processes and coupling of electronic and vibrational excitations are unlikely. We propose the ability of these small clusters to deform after optical excitation being responsible for the short relaxation times analogous to the previously studied case of $\mathrm{Al}_{13}^{-}$. Qualitatively, the relationship of electronic shells and their gaps and the deformation of the clusters is described by the Nilsson diagram. With the help of this diagram, a first qualitative understanding of the experimental data can be gained.

\section{ACKNOWLEDGMENTS}

We acknowledge DFG (Deutsche Forschungsgemeinschaft) and CAP (Center for Applied Photonics, University of Konstanz) for the financial support. 
${ }^{1}$ M. T. Zanni, B. J. Greenblatt, A. V. Davis, and D. M. Neumark, J. Chem. Phys. 111, 2991 (1999).

${ }^{2}$ A. E. Bragg, J. R. R. Verlet, A. Kammrath, O. Cheshnovsky, and D. M. Neumark, J. Chem. Phys. 122, 054314 (2005).

${ }^{3}$ G. Ganteför, S. Kraus, and W. Eberhardt, J. Electron Spectrosc. Relat. Phenom. 88, 35 (1998).

${ }^{4}$ N. Pontius, P. S. Bechthold, M. Neeb, and W. Eberhardt, Phys. Rev. Lett. 84, 1132 (2000).

${ }^{5}$ N. Pontius, M. Neeb, W. Eberhardt, G. Lüttgens, and P. S. Bechthold, Phys. Rev. B 67, 035425 (2003).

${ }^{6}$ P. Gerhardt, M. Niemietz, Y. D. Kim, and G. Ganteför, Chem. Phys. Lett. 382, 454 (2003).

${ }^{7}$ M. Niemietz, P. Gerhardt, G. Ganteför, and Y. D. Kim, Chem. Phys. Lett. 380, 99 (2003).

${ }^{8}$ Y. D. Kim, M. Niemietz, P. Gerhart, F. v. Gynz-Rekowski, and G. Ganteför, Phys. Rev. B 70, 035421 (2004).

${ }^{9}$ R. Haight, Surf. Sci. Rep. 21, 275 (1995).

${ }^{10}$ E. Knoesel, A. Hotzel, T. Hertel, M. Wolf, and G. Ertl, Surf. Sci. 368, 76 (1996).

${ }^{11}$ M. Wolf, Surf. Sci. 377, 343 (1997).

${ }^{12}$ M. Aeschlimann, M. Bauer, and S. Pawlik, Chem. Phys. 205, 127 (1996).

${ }^{13}$ R. D. Schaller, J. M. Pietryga, S. V. Goupalov, M. A. Petruska, S. A. Ivanov, and V. I. Klimov, Phys. Rev. Lett. 95, 196401-1 (2005).

${ }^{14} \mathrm{~J}$. Michl and V. Bonacic-Koutecky, Electronic Aspects of Organic Photochemistry (Wiley, New York, 1990).

${ }^{15}$ M. Klessinger and J. Michl, Excited States and Photochemistry of Organic Molecules (VCH, New York, 1995).

${ }^{16}$ A. Kühl and W. Domcke, J. Chem. Phys. 116, 263 (2002).

${ }^{17}$ V. V. Kresin and Y. N. Ovchinnikov, Phys. Rev. B 73, 115412 (2006)

${ }^{18}$ H. Handschuh, X. Chia-Yen Cha, H. Möller, P. S. Bechthold, G. Ganteför, and W. Eberhardt, Chem. Phys. Lett. 227, 496 (1994).

${ }^{19}$ W. A. de Heer, Rev. Mod. Phys. 65, 611 (1993).
${ }^{20}$ J. Ho, K. M. Ervin, and W. C. Lineberger, J. Chem. Phys. 93, 6987 (1990).

${ }^{21}$ K. J. Taylor, C. L. Pettiette-Hall, O. Cheshnovsky, and R. E. Smalley, J. Chem. Phys. 96, 3319 (1992).

${ }^{22}$ H. Handschuh, C.-Y. Cha, P. S. Bechthold, G. Ganteför, and W. Eberhardt, J. Chem. Phys. 102, 6406 (1995).

${ }^{23}$ S. Wolf, G. Sommerer, S. Rutz, E. Schreiber, T. Leisner, L. Wöste, and R. S. Berry, Phys. Rev. Lett. 74, 4177 (1995).

${ }^{24}$ F. Federmann, K. Hoffmann, N. Quaas, and J. P. Toennies, Eur. Phys. J. D 9, 11 (1999).

${ }^{25}$ C. Félix, C. Sieber, W. Harbich, J. Buttet, I. Rabin, W. Schulze, and G. Ertl, Phys. Rev. Lett. 86, 2992 (2001).

${ }^{26}$ P. Radcliffe, A. Przystawik, T. Diederich, T. Döppner, J. Tiggesbäumker, and K.-H. Meiwes-Broer, Phys. Rev. Lett. 92, 173403 (2004).

${ }^{27}$ G. Ganteför, D. M. Cox, and A. Kaldor, J. Chem. Phys. 93, 8395 (1990).

${ }^{28}$ We distinguish between lifetime of an excited state and the relaxation time of a cluster. The lifetime of an excited state is given by an exponential factor describing the depopulation of the state independent of the decay channel. As relaxation time we regard the time the system needs to relax back into the ground state corresponding to the vanishing of any time-dependent signal in the photoelectron spectrum. Usually, the relaxation of an excited state proceeds via various steps in a cascade and the lifetime is the first step of such a cascade. Since we usually cannot distinguish between different decay steps within a cascade on the base of our photoelectron data alone, we can only determine the relaxation time, which contains rather limited information about the dynamics of the system.

${ }^{29}$ H. Häkkinen, M. Moseler, and U. Landman, Phys. Rev. Lett. 89, 033401 (2002).

${ }^{30}$ F. Furche, R. Ahlrichs, P. Weis, C. Jacob, S. Gilb, T. Bierweiler, and M. M. Kappes, J. Chem. Phys. 117, 6982 (2002). 